\title{
LA ESCUELA EN LA ERA DIGITAL: SMARTPHONES, APPS Y PROGRAMACIÓN EN EDUCACIÓN PRIMARIA Y SU REPERCUSIÓN EN LA COMPETENCIA MEDIÁTICA DEL ALUMNADO
}

\section{SCHOOL IN THE DIGITAL AGE: SMARTPHONES, APPS AND PROGRAMMING IN PRIMARY EDUCATION AND ITS INFLUENCE IN THE STUDENT'S DIGITAL AND MEDIA SKILLS}

\author{
Mónica Bonilla-del-Río ${ }^{1}$ \\ monica.bonilla@alumnos.unican.es \\ Dr. Ignacio Aguaded ${ }^{2}$ \\ ignacio@aguaded.es

\begin{abstract}
${ }^{1}$ Universidad de Cantabria. Facultad de Educación. Departamento de Educación. Avd. Los Castros, s/n, 39005, Santander (España) Carmen. 21071, Huelva (España)
\end{abstract} \\ ${ }^{2}$ Universidad de Huelva. Facultad de Educación. Departamento de Educación. Campus El
}

Resumen: La sociedad actual caracterizada por fenómenos como la globalización, el entorno multipantalla o los continuos progresos científicos y tecnológicos está provocando modificaciones constantes a nivel económico, social, académico y cultural. Estos cambios configuran nuevos escenarios y retos pedagógicos a los que la escuela debe adaptarse. Desde las instituciones educativas surge la necesidad de promover la alfabetización mediática de los ciudadanos, especialmente de los niños y jóvenes. Este trabajo recoge los resultados obtenidos en una investigación realizada en un colegio de la Comunidad Autónoma de Cantabria, con el fin de analizar el uso que hacen de los dispositivos tecnológicos los niños de Educación Primaria. Asimismo, se pretende profundizar sobre la inclusión de las TIC en las aulas y conocer las percepciones de los diferentes agentes de la comunidad educativa (docente, familias y estudiantes). Los resultados muestran que el alumnado es capaz de realizar diversas tareas a través de las TIC y que los diferentes agentes educativos valoran positivamente los proyectos que se desarrollan en el centro. Se concluye el trabajo destacando la posibilidad de complementar estas experiencias con prácticas que fomenten la competencia mediática y no únicamente la digital, con el fin de formar ciudadanos responsables $y$ críticos ante los medios.

Palabras clave: Alfabetización mediática, alfabetización digital, Educación Primaria, TIC.

Abstract: Modern society characterized by phenomena such as globalization, multi-screen world or continuous scientific and technological progress, is causing constant economic, social, academic and cultural changes. These changes define new pedagogical scenarios and challenges and school must adapt to them. From the educational institutions emerges the need to promote media literacy of citizens, especially children and young people. This paper discusses the results obtained in a research carried out in a school in Cantabria, in order to analyze the use of technological devices by Primary Education children. Furthermore, it also explores the use of ICT in classrooms and the perceptions of different agents of the educational community (teacher, families and students). Results show that students can do various tasks through ICT and that the different educational agents consider the projects that are developed in this school as positive experiences. The paper concludes highlighting the possibility to complementing these experiences with practices that promote media skills, not just digital, in order to form responsible and critical citizens with media.

Keywords: Media literacy, digital literacy, Primary Education, ICT.

Píxel-Bit. Revista de Medios y Educación. No 53 Julio 2018. ISSN: 1133-8482. e-ISSN: 2171-7966. doi: http://dx.doi.org/10.12795/pixelbit.2018.i53.10 


\section{Introducción}

Es innegable que hoy en día la alfabetización mediática es un factor fundamental e indispensable en la sociedad actual que permite promover la participación ciudadana y la inclusión social, siendo considerada como un derecho básico en los sistemas democráticos (Avello et. al., 2013). La escuela no debe ser ajena a las transformaciones que las TIC están provocando a nivel personal, profesional, académico y social y ha de adaptarse a las nuevas formas de aprender y acceder al conocimiento de las nuevas generaciones (Pérez-Escoda, Castro-Zubizarreta y FandosIgado, 2016).

Los niños crecen y se desarrollan en contextos repletos de recursos tecnológicos, como acuñó Prensky (2001), son nativos digitales. Sin embargo, el mero hecho de que los niños y jóvenes convivan con este tipo de dispositivos y medios no garantiza que hagan un uso adecuado y responsable de los mismos ni que a través de su uso mejoren sus competencias

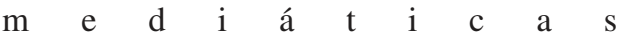
(Pérez-Tornero \& Martínez-Cerdá, 2011; Sánchez-Carrero \& Aguaded, 2013; Ramírez García \& González-Fernández, 2016). Por tanto, surge la necesidad de educar en medios, con el fin de formar ciudadanos críticos y responsables (Aguaded \& Guerra, 2012).

La UNESCO mediante el «Currículum para Profesores sobre Alfabetización Mediática e Informacional (AMI)» pone de manifiesto la necesidad de promover la formación de los docentes en este ámbito, con el fin de que puedan transmitir a los alumnos habilidades que les permitan «aprender a aprender»y participar en la sociedad como ciudadanos racionales e informados.

Según Wilson, Grizzle, Tuazon, Akyempong y Cheung (2011), la alfabetización digital hace referencia a la habilidad de hacer uso de la tecnología digital para buscar, interpretar, utilizar y producir información, así como entender y usar dicha información en diversos formatos, aplicando el conocimiento obtenido en entornos digitales. Para ello, además de tener acceso a internet y a recursos técnicos, es de vital importancia la adquisición de estrategias que permitan al individuo encontrar y procesar la información, valorando si es valiosa, adecuada y confiable (Maglione \& Varlotta, 2012). Por otra parte, la alfabetización mediática es la habilidad para decodificar, examinar, valorar y producir contenidos comunicativos, comprendiendo y haciendo uso de los medios de comunicación de manera segura, crítica y responsable (Wilson et al., 2011). Tanto el Parlamento como la Comisión Europea consideran necesario fomentar la educación en medios, analizando y difundiendo buenas prácticas desde los contextos educativos. De acuerdo con las Recomendaciones de la Comisión Europea (2009), la alfabetización mediática se ha convertido en un requisito previo para alcanzar una ciudadanía plena y activa y una manera de prevenir y reducir el riesgo de exclusión social. En este sentido, la Ley Orgánica 8/2013, de 9 de diciembre, para la mejora de la calidad educativa, vigente en la actualidad, destaca la necesidad de adecuación de las metodologías a las necesidades, características e intereses del alumnado, haciéndoles protagonistas y agentes activos en el proceso de enseñanza aprendizaje. De esta manera, la legislación hace especial incidencia en la inclusión de las Tecnologías de la Información y la 
Comunicación (TIC) en la escuela, con el fin de conectar con las experiencias y hábitos de las nuevas generaciones, adaptar las prácticas didácticas a las necesidades y el ritmo de cada alumno y ofrecerles una atención más personalizada. Por consiguiente, las TIC son consideradas como un elemento fundamental para la transformación del sistema educativo. Teniendo en cuenta las recomendaciones del Parlamento Europeo, el Real Decreto, 126/ 2014, de 28 de febrero, por el que se establece el currículo básico de la Educación Primaria, fundamenta la potenciación del aprendizaje por competencias a través de su integración en los elementos curriculares. Se identifican siete competencias clave, entre las que se encuentra la «competencia digital». Este hecho implica el reconocimiento curricular a la importancia de alfabetizar en este ámbito, introduciendo las tecnologías en las aulas para adaptar la educación a las necesidades de la sociedad actual. Hoy en día es necesario apostar por el desarrollo de la competencia de «aprender a aprender» y de las denominadas «e-competencias», es decir, potenciar aquellas destrezas que requiere el ciudadano conectado entre las que destacan el trabajo colaborativo, las habilidades de búsqueda y filtrado de información fiable y de calidad, la creatividad o la capacidad de síntesis (Reig \& Vílchez, 2013).

En este sentido, Sánchez y Castro (2014) señalan que los nativos digitales se caracterizan por la habilidad y desenvoltura para acceder a la información en la red, la adaptación a las novedades tecnológicas de manera ágil e inmediata y el gusto y el disfrute por el aprendizaje lúdico, cooperativo, en red y a través de métodos interactivos. Del mismo modo, los nativos digitales, de acuerdo con Feijoo-Fernández y García-González (2017), tienen la habilidad de prestar atención a varios aspectos al mismo tiempo y de forma rápida, no procesan la información de manera secuencial, muestran preferencia por las imágenes por encima del texto y sus procesos de lecturas no son lineales sino aleatoriamente guiados y personalizados a través de los hipertextos.

Sin embargo, De la Torre (2009) apunta que, a pesar de las habilidades para el uso de recursos digitales, se debería llegar a la desmitificación de que los niños y jóvenes tienen las competencias necesarias y están bien preparados para desenvolverse adecuadamente en el ambiente digital, con el fin de ofrecerles medios, procesos y entornos que les permitan una verdadera alfabetización digital y mediática. Asimismo, es necesario tener en cuenta que los procesos educativos tienen lugar en diversos ámbitos, por lo que es necesario que exista continuidad y coherencia en los aprendizajes que tienen lugar en todos los contextos donde crecen los niños. Por tanto, es de vital importancia que existan procesos de colaboración entre los diferentes agentes educativos, lo que supondría una mejora significativa en la educación de los menores (Bolívar, 2006). El reto educativo consiste, por tanto, en mejorar la relación entre la familia y la escuela, con el fin de construir un proyecto educativo común y de fomentar el desarrollo integral del niño (García, Gomáriz, Hernández \& Parra, 2010).

La función de los docentes no ha de limitarse a la mera transmisión de conocimientos (Prieto, 2008), sino que debe adaptarse a las transformaciones de la sociedad y plantear modelos de aprendizaje a través de estrategias metodológicas que faciliten la inclusión de las TIC en las aulas (Robizo \& Cózar, 2015). Sin embargo, conviene tener en cuenta que la innovación 
tecnológica no garantiza la innovación pedagógica y que la mera presencia de las TIC en las aulas no provoca directamente transformaciones en los procesos de enseñanza-aprendizaje, ya que en muchas ocasiones se emplean los dispositivos digitales para continuar promoviendo modelos tradicionales basados en la enseñanza de tipo expositivo y el aprendizaje pasivo, memorístico y reproductivo (Area, 2015). En este sentido, según Paredes (2010), la figura del docente adquiere gran relevancia, puesto que ha de ser capaz de innovar, reflexionar y transformar sus propuestas didácticas para responder a las demandas sociales a la vez que se alcanzan los objetivos curriculares.

\section{Método}

Durante el desarrollo de esta investigación, centrada en un estudio de casos, se ha utilizado una metodología con un enfoque mixto, aplicándose tanto instrumentos cuantitativos como cualitativos. Se ha optado por la triangulación de instrumentos de recogida de información (cuestionarios, entrevista y grupo de discusión) y por la triangulación de muestras (docente, familias y alumnado), con el propósito de ofrecer una respuesta holística y global del objeto de estudio.

En este sentido, se ha aplicado un primer cuestionario al alumnado del sexto curso de Educación Primaria del CEIP Elena Quiroga, situado en la localidad de Peñacastillo (Cantabria). En este caso, se ha empleado el cuestionario propuesto por Pérez-Escoda (2015), cuya finalidad es conocer la competencia digital del alumnado, debido a que está dirigido a estudiantes de esta etapa educativa y a que ha sido previamente validado. El cuestionario divide las variables de estudio en dos categorías. Por un lado, se definen las variables de contexto, que incluyen aquellas preguntas relacionadas con la información demográfica de los participantes y, por otra parte, las variables de criterio que se dividen en los siguientes indicadores: Uso de las TIC y frecuencia de uso en entornos informales; Grado de integración de las TIC en las actividades cotidianas; Capacidad para realizar tareas relacionadas con la competencia digital. No obstante, se ha optado por ampliar dicho instrumento con la generación de cinco ítems relacionados con la competencia mediática que complementan la herramienta. Para ello, se ha recurrido a una fuente bibliográfica reconocida a nivel internacional como es el «Kit de medios para la alfabetización audiovisual» de Share, Jolls y Thoman (2006), en el que se plantea un marco de referencia para aprender y enseñar en la era mediática. Esto ha derivado en un nuevo indicador: Capacidad para realizar tareas relacionadas con la competencia mediática. Por tanto, a través de este instrumento se pretende conocer el uso que hacen los estudiantes de los recursos tecnológicos, así como analizar las tareas que son capaces de realizar en función de sus competencias digitales y mediáticas. Dicho cuestionario fue respondido por cuarenta y cuatro alumnos.

Posteriormente, se ha aplicado otro cuestionario dirigido a las familias del alumnado del sexto curso del centro citado anteriormente. El objetivo de esta herramienta es conocer la valoración que hacen los progenitores sobre la inclusión de las TIC en las aulas y especialmente sobre el proyecto de programación y robótica que se desarrolla en este colegio. Para la validación de este instrumento se ha recurrido al juicio de 


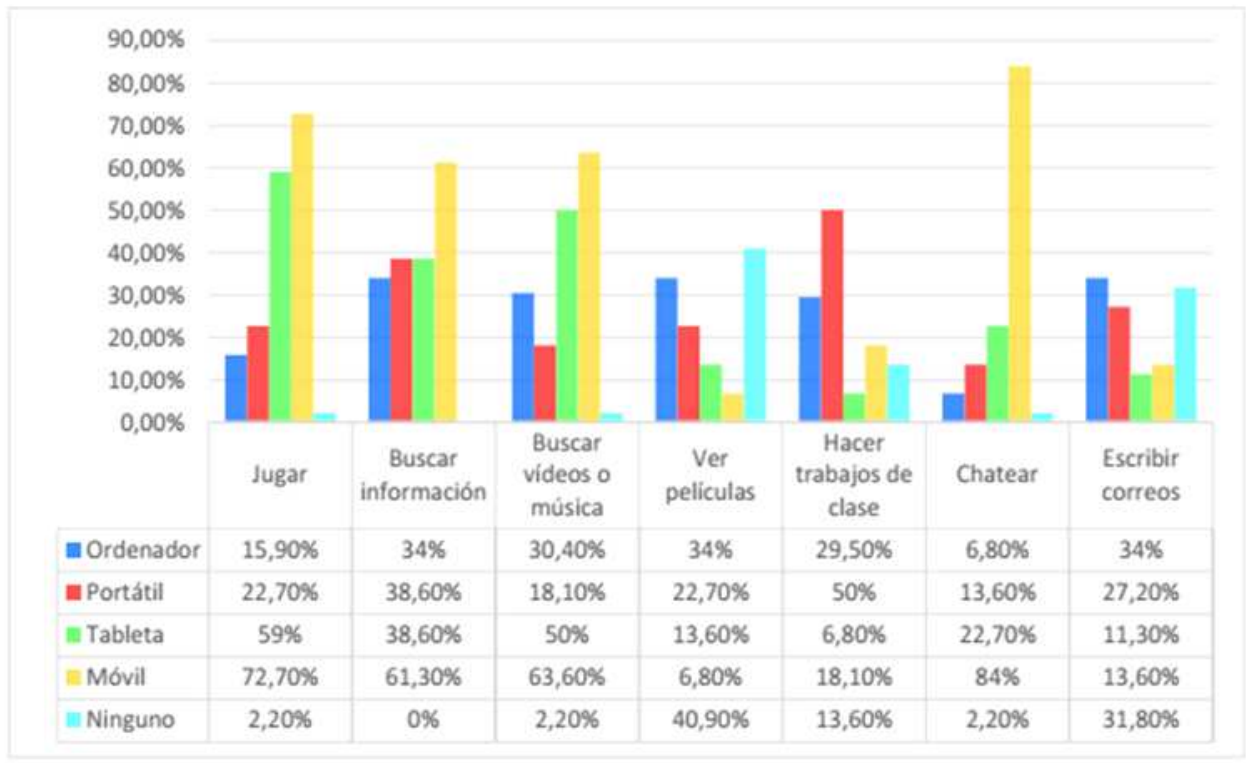

Figura 1. Porcentajes del alumnado que emplea los dispositivos en función de las tareas.

expertos, tras cuyo análisis se depuraron las preguntas iniciales, eliminando sesgos como la deseabilidad social o las preguntas que pudieran generar diferentes interpretaciones. Un total de veinticinco padres respondieron al cuestionario.

Asimismo, se realizó una entrevista al maestro de sexto que imparte los contenidos relacionados con la programación y la robótica educativa para conocer su opinión sobre la inclusión de las TIC en la escuela. El instrumento fue sometido a un proceso de validación de expertos.

Del mismo modo, se ha llevado a cabo un grupo de discusión con algunos alumnos, con el fin de conocer su punto de vista y profundizar sobre cómo conciben la realidad educativa. Este instrumento también fue sometido a la validación de expertos, cuyas consideraciones y aportaciones sirvieron para modificar y reajustar algunas de las preguntas iniciales. Se seleccionó de manera aleatoria a seis alumnos de sexto para la participación en el grupo de discusión.

\section{Resultados}

En primer lugar, se muestran los datos obtenidos en el cuestionario dirigido al alumnado. En función del primer indicador Uso de las TIC y frecuencia de uso en entornos informales los resultados revelan que el dispositivo que más utilizan los niños es el smartphone, ya que el $82 \%$ de los alumnos declaran hacer uso de este recurso, seguido de la tablet (61\%). Resulta significativa la escasa utilización que manifiestan los niños en relación con el uso del ordenador (27\%) o el portátil (36\%), ya que menos de la mitad de los encuestados 


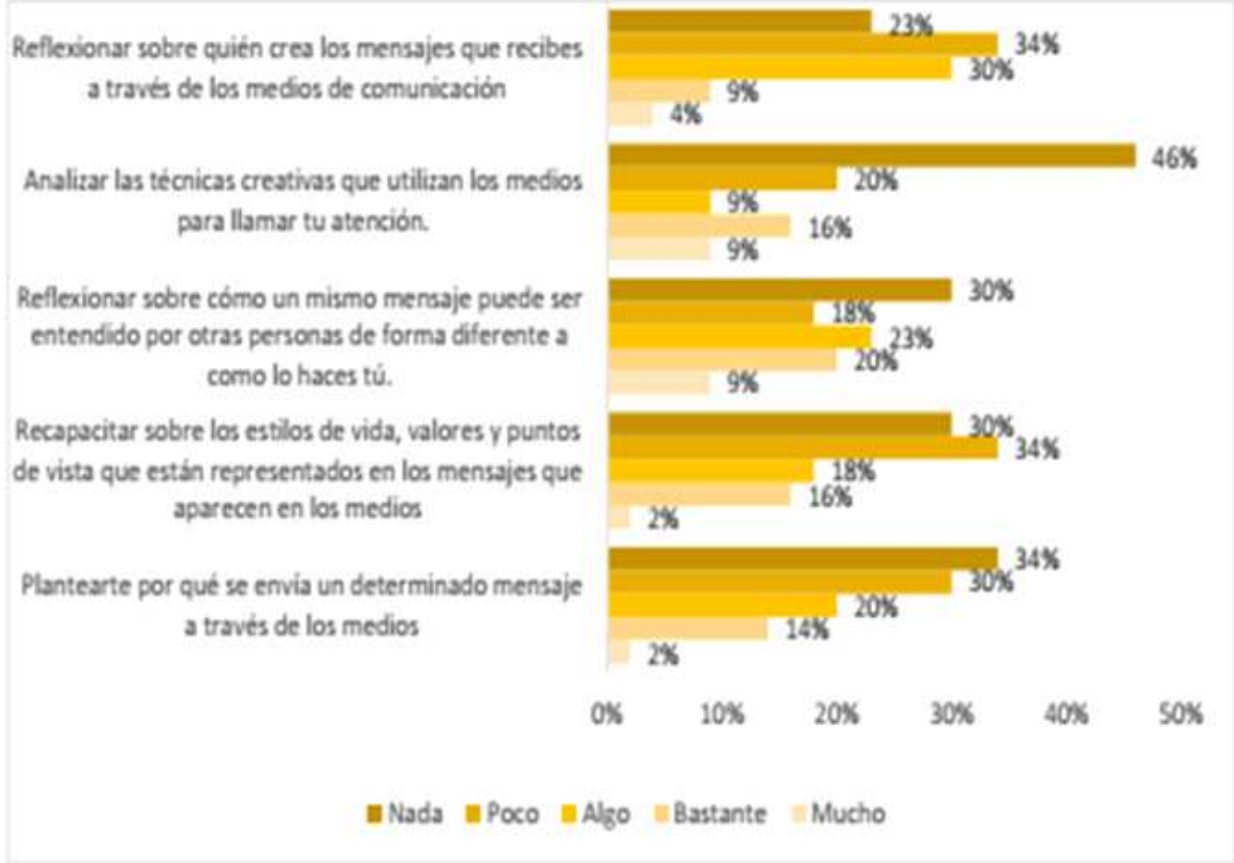

Figura 2. Frecuencias de las tareas realizadas.

hacen uso de estos dispositivos. Por otra parte, el $64 \%$ de los niños manifiesta que suele usar más de un dispositivo, mientras que el $36 \%$ de los estudiantes declara que suele hacer uso de un único dispositivo. Todos los alumnos encuestados se conectan a Internet y más de la mitad se conecta a la Red casi todos los días (66\%). El porcentaje de los estudiantes que casi no se conecta a Internet es eminentemente bajo, lo que pone de relieve que los niños encuestados acceden a Internet con bastante asiduidad.

En lo referente al segundo indicador Grado de integración de las TIC en las actividades cotidianas, en la figura 1 se muestra el porcentaje de alumnado que hace uso de los dispositivos en función de las actividades que realiza con los diferentes aparatos tecnológicos.
En cuanto a la frecuencia en la que los niños manifiestan que utilizan los dispositivos, los datos muestran que hay tres actividades a las que al menos la mitad de los alumnos encuestados dedican tiempo casi todos los días: chatear con amigos (61\%), jugar (54\%) y buscar vídeos o música (50\%). En contraposición, las tareas a las que menos tiempo dedican son: hacer trabajos académicos, ver películas y escribir correos electrónicos.

Los datos en función del tercer indicador Capacidad para realizar tareas relacionadas con la competencia digital pretenden determinar el dominio digital de los estudiantes a través de su autopercepción. Los ítems están estrechamente vinculados con estas áreas competenciales: información, comunicación, creación de contenido, 
seguridad y resolución de problemas (PérezEscoda, 2015). Los resultados del área de información revelan que la tarea que más realizan los alumnos en esta categoría es la visualización de vídeos de YouTube seguida de la navegación por internet. En el área de comunicación, los datos muestran que las tareas menos realizadas por los alumnos son el envío de correos electrónicos y el uso de redes sociales mientras que la participación en chats es una práctica bastante extendida entre el alumnado. En cuanto al área competencial relacionada con la creación de contenidos, los datos muestran al menos la mitad de los niños declaran hacer y editar fotos, realizar trabajos académicos o grabar vídeos con cierta frecuencia. En lo referente al área de seguridad, la mayoría de los alumnos manifiesta dar sus datos personales con escasa frecuencia. Por último, en cuanto al área de resolución de problemas los datos revelan que al menos la mitad de los niños intercambia información entre distintos dispositivos, actualiza aplicaciones o reinicia su ordenador, móvil o tablet.

Finalmente, en relación con el indicador Capacidad para realizar tareas relacionadas con la competencia mediática cabe destacar al menos la mitad de los encuestados, declaran reflexionar con ninguna o con poca frecuencia sobre las tareas planteadas. En contraposición, las opciones de respuesta que indicaban bastante y mucha frecuencia adquieren una representación significativamente escasa.

Através del cuestionario dirigido a las familias, se tratará de conocer en primer lugar cuál es la valoración que hacen los familiares sobre el uso de las TIC en el ámbito educativo, así como la importancia que le atribuyen al desarrollo de la competencia digital. En este sentido, el $92 \%$ de los encuestados considera que el uso de las TIC en esta etapa educativa es fundamental y el $88 \%$ manifiesta que es necesario desarrollar la competencia digital desde edades tempranas. Asimismo, un alto porcentaje de las familias considera que las TIC son un elemento motivador para sus hijos en los procesos de aprendizaje.

Por otra parte, los datos muestran que existe un porcentaje próximo entre las familias que conocen los proyectos educativos con TIC que se desarrollan en el centro $(52 \%)$ y las que no (48\%). En este sentido, el 52\% encuestados consideran que la información proporcionada por los docentes sobre estos proyectos es suficiente y relevante, aunque el $48 \%$ restante declara que no le parece bastante o útil $(24 \%)$ o que no sabe si se proporciona este tipo de información (24\%). Al 76\% de los encuestados les gustaría que existiese una propuesta dirigida a las familias de formación en TIC impartida en el propio centro educativo y ante una hipotética realización de un taller sobre programación y robótica para familias, el $68 \%$ de los participantes manifiesta que participaría frente a un $20 \%$ que declara que no lo realizaría y un $12 \%$ que indica no saber si participaría.

En relación con los hábitos y actitudes de los hijos ante el uso de las TIC, el $84 \%$ de los padres declara hablar con sus hijos sobre la utilización de las TIC en el ámbito escolar frente a un $16 \%$ que señala que no suele hablar con su hijo sobre este tipo de cuestiones. Al $60 \%$ de los familiares les gusta que su hijo emplee el smartphone como una herramienta más de aprendizaje, aunque tan solo el $28 \%$ de los encuestados señala que les agrada que sus hijos tengan que llevar el móvil al colegio, aunque sea con fines didácticos. En contraposición, el 52\% de los padres afirma 
que no le gusta que tenga que llevar el dispositivo al centro y el $20 \%$ no lo sabe.

Por último, cabe destacar que donde si existe unanimidad es en la consideración de las TIC como un elemento motivador para los alumnos. En este sentido, el $96 \%$ de los participantes exponen que su hijo muestra actitudes positivas hacia el aprendizaje cuando hace uso de las TIC.

En función del conocimiento sobre algunos recursos tecnológicos, los datos revelan que un porcentaje elevado de las familias (84\%) declara que conoce los recursos TIC con los que trabaja su hijo en el colegio. No obstante, al hacer referencia a recursos o entornos virtuales específicos, los padres manifiestan no tener conocimiento de este tipo de herramientas. De los recursos mencionados en el cuestionario, «Scratch» es el más reconocido por los padres. Sin embargo, tan solo el $40 \%$ indica que lo conoce. En cuanto al dominio de esta plataforma, el $88 \%$ de los padres declara no saber utilizarla. Por otra parte, el $76 \%$ de los padres indica que desconoce el recurso «App Inventor» frente a un $20 \%$ que lo conoce. No obstante, casi la totalidad de los encuestados (92\%) indican que no tienen dominio para hacer uso de este recurso. Por último, el $28 \%$ de $\operatorname{los}$ participantes considera que es interesante la utilización del kit de robótica LEGO WeDO para el aprendizaje de los niños. Tan solo el $4 \%$ manifiesta que no es un recurso interesante y el $68 \%$ lo desconoce.

Finalmente, en lo referente a la organización curricular de las experiencias pedagógicas con TIC, los datos muestran que un alto porcentaje de los padres (88\%) expone que la introducción de contenidos sobre programación y robótica en la Educación Primaria es interesante. Asimismo, el 88\% también considera que es adecuado que exista un horario flexible y que se empleen los desdobles para la realización de este tipo de proyectos. En esta línea, el $72 \%$ de los progenitores manifiesta que prefiere que se lleven a cabo actividades de programación y robótica, frente a un $16 \%$ de padres que preferiría que no se desarrollasen este tipo de proyectos. Ante la hipotética propuesta de introducción de una asignatura específica que abordase este tipo de contenidos, el 64\% de la muestra declara que le resultaría interesante mientras que el $12 \%$ se muestra en desacuerdo y el $24 \%$ desconoce si sería adecuado. Por otra parte, el $56 \%$ de los padres considera que este tipo de contenidos deberían trabajarse de manera transversal, es decir, desde diferentes asignaturas frente al $4 \%$ que está en desacuerdo con este planteamiento y el $40 \%$ que lo desconoce. No obstante, suele haber bastante unanimidad ante la propuesta de impartir contenidos de programación y robótica únicamente en actividades extraescolares. En este caso, el $79 \%$ de los padres declaran que no debe trabajarse solamente fuera del horario lectivo, mientras que el $7 \%$ considera que sí deberían ser contenidos extraescolares y el $14 \%$ lo desconoce.

En cuanto al grupo de discusión, cabe destacar que en general los alumnos otorgan gran importancia a la utilización de las TIC en las aulas. Las principales razones que alegan son la motivación ante el uso de este tipo de recursos y la necesidad de ser competentes digitalmente en el futuro. La valoración de los niños sobre los proyectos TIC realizados en el centro es ciertamente favorable, ya que manifiestan mostrar interés y actitudes positivas hacia el aprendizaje en este ámbito. Sin embargo, también son conscientes de sus posibilidades y reconocen sus limitaciones a la hora de emplear algunos recursos para la 
creación de contenidos audiovisuales. Respecto la utilización del smartphone como una herramienta más de aprendizaje, los resultados obtenidos muestran diferentes puntos de vista. Algunos alumnos consideran que son recursos motivantes y más accesibles y cómodos en comparación con los portátiles, pero otros manifiestan que pueden ser elementos distractores.

También declaran que la metodología docente es adecuada y valoran positivamente las actividades planteadas por el profesor. En este sentido, consideran la creación de videotutoriales por parte del maestro como un elemento enriquecedor. Destacan que es conveniente el planteamiento de las clases porque tiene continuidad y no son prácticas aisladas o esporádicas. Sin embargo, indican posibles mejoras en relación a una mayor flexibilidad en cuanto al diseño y el proceso creativo de los recursos audiovisuales por parte de los propios niños. La visión del alumnado con respecto al nivel de competencia digital de sus familias es negativa. Los niños señalan que, en general, sus padres no están preparados y no disponen de la formación suficiente para ayudarles si tienen alguna dificultad en el uso de las TIC. La totalidad de los alumnos muestra actitudes positivas ante la inclusión de la programación y la robótica en las escuelas y consideran que son contenidos interesantes que deberían tratarse en el currículum obligatorio.

Por último, en cuanto a la entrevista al docente, conviene mencionar que el maestro destaca la utilización del Kit de robótica «LEGO WeDo», la iniciación con los Makeblock, la introducción de Google Classroom, el trabajo en torno a Windows, el programa de computación creativa »Scratch 2.0" y la plataforma «App Inventor».
Asimismo, el profesor manifiesta la necesidad de incluir las TIC en las metodologías de la etapa de Educación Primaria y alega que la legislación hace hincapié tanto a nivel estatal como autonómico a la importancia de desarrollar habilidades informacionales y audiovisuales en los niños, promoviendo su competencia digital. En este sentido, destaca la realización del proyecto sobre programación y robótica como una experiencia enriquecedora. No obstante, señala que la implicación del profesorado no es ciertamente efectiva y apunta a que existen diferencias significativas ante el uso que los docentes hacen de los recursos tecnológicos. El maestro señala la importancia de conocer los intereses del alumnado y los recursos que utilizan, con el fin de poder dar respuestas educativas que se adapten a las necesidades de la sociedad actual. La percepción del docente ante la actitud que muestran los estudiantes hacia el aprendizaje a través de las TIC es positiva. En este sentido, el maestro manifiesta que los niños suelen mostrar altos niveles de satisfacción ante las tecnologías y los proyectos que se han realizado en el centro. Asimismo, el profesor expone que la robótica y, concretamente, los LEGOS son recursos que a los alumnos les parecen atractivos, por lo que considera que tienen gran potencialidad educativa. Además, declara que el trabajo a través de las TIC favorece el aprendizaje, al ser un elemento motivador para los estudiantes. Por último, indica las posibilidades de recursos como «Scratch» para la promoción de la creatividad de los estudiantes, ya que este tipo de entornos permite al alumnado diseñar, crear y elaborar recursos audiovisuales en los que ellos mismos han de programar las acciones, movimientos o sonidos que formarán parte de su creación. Este tipo de experiencias 
requieren cierta habilidad y esfuerzo cognitivo, por lo que resulta un recurso valioso para la promoción de la competencia digital del alumnado.

\section{Discusión y conclusiones}

La alfabetización digital y mediática es una necesidad imperiosa en la sociedad actual y el desarrollo de competencias relacionadas con los ámbitos de consumo y producción en torno a los recursos tecnológicos es cada vez más importante.

Los resultados obtenidos mediante los distintos instrumentos permiten concluir que en este colegio se llevan a cabo proyectos que pretenden potenciar el uso de las TIC en las aulas. En este caso, tanto el alumnado como el docente resaltan las experiencias didácticas realizadas en torno a la programación y la robótica. Del mismo modo, conviene destacar que los recursos empleados para este fin son: ordenadores miniportátiles pertenecientes al programa $\begin{array}{llll}\text { Escuela } & 2.0 & \text { de } & \text { los }\end{array}$ que dispone el centro educativo; herramientas de robótica como el «Kit LEGO WeDo» o los «Makeblock»; Pantallas Digitales Interactivas (PDI); o los smartphones personales de los propios alumnos que llevan al colegio con fines didácticos. En cuanto a los programas que se utilizan cabe destacar la plataforma «Scratch $2.0 »$ y el recurso «App Inventor» a través de los cuales se proponen experiencias educativas como la creación y programación de videojuegos o cuentos audiovisuales, el diseño y creación de aplicaciones para smartphones o tablets con sistema Android o la iniciación en prácticas con robótica educativa. Este tipo de propuestas se realizan con los grupos desdoblados y con frecuencia semanal, con el propósito de alcanzar un mayor aprovechamiento de los recursos disponibles y de prestar una atención más individualizada a los estudiantes.

Asimismo, los resultados obtenidos permiten concluir que los alumnos realizan diversas tareas en su vida cotidiana relacionadas con el uso de las tecnologías, con una frecuencia que varía en función de los dispositivos empleados o la actividad planteada. Cabe destacar que la realización de tareas a través del smartphone entre los niños que componen la muestra es una práctica bastante extendida y generalizada. También resulta significativa la navegación de los niños en Internet, ya que casi la totalidad del alumnado se conecta a Internet al menos una vez por semana. Es necesario, por tanto, incluir en las escuelas contenidos que fomenten el uso de las TIC y la navegación online segura y crítica de los niños, con el fin de ayudarles a evitar posibles riesgos y a aprovechar las potencialidades que ofrecen las TIC.

Sin embargo, a pesar de que las TIC están integradas en su cotidianeidad y del cierto dominio que tienen en el uso de estos recursos, los resultados obtenidos en los ítems relativos a la competencia mediática son menos esperanzadores, ya que un porcentaje significativamente bajo del alumnado reflexiona sobre los mensajes que se transmiten en los medios. Estos datos evidencian que los alumnos han desarrollado más las competencias digitales que las relacionadas directamente con la competencia mediática. Es necesario, por lo tanto, valorar la importancia de promover el desarrollo del pensamiento crítico y reflexivo desde edades tempranas y replantearse las prácticas didácticas que se llevan a cabo en las escuelas, con el fin de fomentar prácticas que no se 
limiten al desarrollo de habilidades instrumentales, sino que busquen el verdadero uso efectivo de los recursos y el consumo crítico ante los medios (Arrieta \& Montes, 2011). En este sentido, podría resultar ciertamente interesante la posibilidad de plantearse incluir alguna propuesta didáctica que complementase las experiencias que se desarrollan actualmente en el centro educativo para enriquecer la formación del alumnado en este ámbito.

En esta línea, conviene mencionar que a través de la triangulación se puede concluir que todos los participantes consideran que las prácticas que desarrollan en el CEIP Elena Quiroga en torno a las TIC son pertinentes y necesarias, ya que tanto el maestro como los estudiantes y las familias destacan la relevancia de desarrollar las competencias digitales y la importancia de que la escuela se adapte a las necesidades de la sociedad actual. Asimismo, todos los agentes educativos coinciden en que este tipo de contenidos deben formar parte del currículum obligatorio y trabajarse durante el horario lectivo. Este dato resulta significativo, ya que demuestra la importancia que otorgan a la alfabetización digital y la necesidad de que estos contenidos no se trabajen únicamente en actividades extraescolares.

Otro a aspecto a destacar es el interés que manifiestan las familias ante la propuesta de recibir formación en TIC en el centro educativo, es decir, los padres reclaman la participación en actividades de formación para mejorar sus competencias digitales y mediáticas. Sería interesante que la escuela abriese sus puertas y atendiese a estas demandas, ya que este proceso formativo supondría una experiencia enriquecedora que permitiría además forjar lazos de colaboración entre la escuela y la familia, lo que supondría la adopción de enfoques cooperativos que favorecerían las relaciones de todos los agentes de la comunidad educativa.

\section{Referencias bibliográficas}

Aguaded, I., \& Guerra, S. (2012). Razones para una educación mediática en la sociedad multipantallas. Sphera Pública, (12), 21-39.

Area, M. (2015). Reinventar la escuela en la sociedad digital. Del aprender repitiendo al aprender creando. En M, Poggi (Coord), Mejorar los aprendizajes en la educación obligatoria. Políticas y actores (167-194). Recuperado de https://goo.gl/2a9Nzn

Arrieta, A., \& Montes, V. D. (2011). Alfabetización digital: uso de las TIC más allá de una formación instrumental y una buena infraestructura. Revista Colombiana de Ciencia Animal, 3(1), 180-197. Recuperado de https://goo.gl/UQ7LSY

Avello, R., López, R., Cañedo, M., Álvarez, H., Granados, J., \& Obando, F. (2013). Evolución de la alfabetización digital: nuevos conceptos y nuevas alfabetizaciones. Medisur, 11(4), 450-457. Recuperado de https:/ /goo.gl/KXcPrN

Bolívar, A. (2006). Familia y escuela: dos mundos llamados a trabajar en común. Revista de educación, 339, 119-146. Recuperado de https://goo.gl/ch8MS5

Comisión Europea (Ed.) (2009). Recomendación de la Comisión, 20 de agosto de 2009, sobre la alfabetización mediática en el entorno digital para una industria audiovisual y de contenidos más competitiva y una sociedad del conocimiento incluyente. Publicada en Diario Oficial de la Unión Europea L 277, del 28 de agosto de 2009. Recuperado de https://goo.gl/1XOBq1

De la Torre, A. (2009). Nuevos perfiles en el alumnado: la creatividad en nativos digitales 
competentes y expertos rutinarios. RUSC. Universities and Knowledge Society Journal, 6(1), 7-14.

Decreto NO. 126/2014. Decreto por el que se establece el currículo básico de la Educación Primaria. Publicado en Boletín Oficial del Estado. No. 52, del 1 de marzo de 2014. España. Recuperado de https://goo.gl/ 08hQc6

Feijoo-Fernández, B.Y García-González, A. (2017). El entorno del niño en la cultura digital desde la perspectiva intergeneracional. Aposta. Revista de Ciencias Sociales, 72, 9-27. Recuperado de https://goo.gl/L7Z3i2

García, M.P., Gomáriz, M.Á., Hernández, M.Á., \& Parra, J. (2010). La comunicación entre la familia y el centro educativo, desde la percepción de los padres y madres de los alumnos. Educatio siglo XXI, 28(1), 157- 187. Recuperado de https://goo.gl/crqMjH

Ley Orgánica 8/2013, de 9 de diciembre, para la Mejora de la Calidad Educativa. Boletín Oficial del Estado número 295, de 10 de diciembre de 2013.

Maglione, C. \& Varlotta, N. (2012). Investigación, gestión y búsqueda de información en Internet. Colombia: Conectar-Igualdad. Recuperado de https:// goo.gl/DVnE1N

Paredes, J. (2010). Innovadores en espacios reinstrumentalizados. Aproximaciones etnográficas y narrativas a los centros innovadores con TIC en educación primaria y secundaria. Revista Iberoamericana sobre Calidad, Eficacia y Cambio en Educación, 8(1), 45-62. Recuperado de https://goo.gl/ zxqVf9

Pérez-Escoda, A. (2015). Alfabetización digital y competencias digitales en el marco de la evaluación educativa: Estudio en docentes y alumnos de Educación
Primaria en Castilla y León. (Tesis doctoral) Universidad de Salamanca.

Pérez-Escoda, A. Castro-Zubizarreta, A. \& Fandos-Igado, M. (2016). La competencia digital de la Generación Z: claves para su introducción curricular en la Educación Primaria. Comunicar, 24(49). https://doi.org/ 10.3916/C49-2016-07

Pérez-Tornero, J.M. \& Martínez-Cerdá, J.F. (2011). Hacia un sistema supranacional de indicadores mediáticos. Infoamérica, 5, 3957.

Prensky, M. (2001). Nativos e inmigrantes digitales. Adaptación al castellano del texto original «Digital Natives, Digital Immigrants» Institución Educativa SEK. Recuperado de http://goo.gl/8DA7t

Prieto, E. (2008). El papel del profesorado en la actualidad. Su función docente y social. Foro de educación, 6(10), 325-345.

Ramírez-García, A. \& González-Fernández, N. (2016). Competencia mediática del profesorado y del alumnado de educación obligatoria en España. Comunicar, 24(49), 4958. https://doi.org/10.3916/C49-2016-05

Reig, D., \& Vílchez, L. (2013). Los jóvenes en la era de la hiperconectividad: tendencias, claves y miradas. Madrid: Fundación Telefónica.

Robizo, M.J., \& Cózar, R. (2015). Usos y competencias en TIC en los futuros maestros de educación infantil y primaria: hacia una alfabetización real para docentes. Pixel-Bit. Revista de Medios y Educación, (47), 23-39.

Sánchez, A., \& Castro, D. (2014). Cerrando la brecha entre nativos e inmigrantes digitales a través de las competencias informáticas e informacionales. Apertura, 5(2), 6-15. Recuperado de https://goo.gl/5AjcSz.

Sánchez-Carrero, J., \& Aguaded, I. (2013). El grado de competencia mediática 
en la ciudadanía andaluza. Estudios sobre el mensaje periodístico, 19(1), 265-280.

Share, J., Jolls, T., \& Thoman, E. (2006). 5 preguntas claves que pueden cambiar el mundo. Segunda parte: Prácticas CML MediaLit Kit. Center for Media Literacy. Recuperado de https://goo.gl/ FnlUnM

Wilson, C., Grizzle, A., Tuazon, R., Akyempong, K., \& Cheung, C. (2011) Alfabetización Mediática e Informacional. Currículum para profesores. Paris: UNESCO. Recuperado de https://goo.gl/JeGv4b

Fecha de recepción: 11-10-2017

Fecha de evaluación: 27-11-2017

Fecha de aceptación: 27-11-2017 